\title{
Serum Wisteria floribunda agglutinin-positive human Mac-2 binding protein level predicts recurrence of hepatitis B virus-related hepatocellular carcinoma after curative resection
}

\author{
Hye Soo Kim ${ }^{1, *}$, Seung Up Kim ${ }^{1,2,}$, Beom Kyung Kim ${ }^{1,2}$, Jun Yong Park ${ }^{1,2}$, Do Young Kim ${ }^{1,2}$, Sang Hoon Ahn , \\ Kwang-Hyub Han ${ }^{1,2}$, Young Nyun Park ${ }^{3}$, Dai Hoon Han ${ }^{4}$, Kyung Sik Kim ${ }^{4}$, Jin Sub Choi ${ }^{4}$, Gi Hong Choi ${ }^{4}$, and Hyon-Suk Kim ${ }^{5}$ \\ 'Department of Internal Medicine, ${ }^{2}$ Institute of Gastroenterology, ${ }^{3}$ Department of Pathology, ${ }^{4}$ Department of Surgery, ${ }^{5}$ Department of \\ Laboratory Medicine, Yonsei University College of Medicine, Seoul, Korea
}

Background/Aims: To investigate whether serum Wisteria floribunda agglutinin-positive human Mac-2-binding protein (WFA $\left.{ }^{+}-\mathrm{M} 2 \mathrm{BP}\right)$ can predict the recurrence of hepatitis B virus ( $\mathrm{HBV}$ )-related hepatocellular carcinoma $(\mathrm{HCC})$ after curative resection.

Methods: Patients with chronic hepatitis B (CHB) who underwent curative resection for HCC between 2004 and 2015 were eligible for the study. Recurrence was sub-classified as early ( $<2$ years) or late ( $\geq 2$ years).

Results: A total of 170 patients with CHB were selected. During the follow-up period (median, 22.6 months), 64 (37.6\%) patients developed recurrence. In multivariate analyses, WFA ${ }^{+}-\mathrm{M} 2 \mathrm{BP}$ level was an independent predictor of overall (hazard ratio $[H R]=1.490)$, early $(H R=1.667)$, and late recurrence $(H R=1.416)$, together with male sex, des-gamma carboxyprothrombin level, maximal tumor size, portal vein invasion, and satellite nodules (all $P<0.05$ ). However, WFA ${ }^{+}$ M2BP level was not predictive of grade B-C posthepatectomy liver failure. The cutoff value that maximized the sum of sensitivity (30.2\%) and specificity (90.6\%) was 2.14 (area under receiver operating characteristic curve $=0.632, P=0.010$ ). Patients with a WFA ${ }^{+}-M 2 B P$ level $>2.14$ experienced recurrence more frequently than those with a WFA ${ }^{+}-M 2 B P$ level $\leq 2.14$ ( $P=0.011$ by log-rank test), and had poorer postoperative outcomes than those with a WFA ${ }^{+}-M 2 B P$ level $\leq 2.14$ in terms of overall recurrence ( 56.0 vs. $34.5 \%, P=0.047$ ) and early recurrence ( 52.0 vs. $20.7 \%, P=0.001)$.

Conclusions: WFA ${ }^{+}-M 2 B P$ level is an independent predictive factor of HBV-related HCC recurrence after curative resection. Further studies should investigate incorporation of WFA+-M2BP level into tailored postoperative surveillance strategies for patients with CHB. (Clin Mol Hepatol 2020;26:33-44)

Keywords: Hepatitis B; Liver fibrosis; Biomarkers; Hepatocellular carcinoma; Prognosis

\begin{abstract}
Abbreviations:
AFP, alpha-fetoprotein; $A \cup C$, area under receiver operating characteristic curve; BCLC, Barcelona Clinic Liver Cancer; CHB, chronic hepatits $\mathrm{B}$; Cl, confidence interval; $\mathrm{COI}$, cutoff index; DCP, des-gamma carboxy prothrombin; $\mathrm{HBV}$, hepatitis B virus; HCC, hepatocellular carcinoma; HR, hazard ratio; ICG R15, indocyanine green retention rate at 15 minutes; INR, international normalized ratio; IQR, interquartile range; $\mathrm{PHLF}$, posthepatectomy liver failure; $\mathrm{ROC}$, receiver operating characteristic; WFA - M2BP, Wisteria floribunda agglutinin-positive human Mac-2 binding protein

*Hye Soo Kim and Seung Up Kim contributed equally to this work.
\end{abstract}

Corresponding author: Gi Hong Choi

Department of Surgery, Yonsei University College of Medicine, 50-1 Yonsei-ro, Seodaemun-gu, Seoul 03722, Korea

Tel: +82-2-2228-2099, Fax: +82-2-313-8289

E-mail:choigh@yuhs.ac

https://orcid.org/0000-0002-1593-3773

\section{Hyon-Suk Kim}

Department of Laboratory Medicine, Yonsei University College of Medicine, 50-1 Yonsei-ro, Seodaemun-gu, Seoul 03722, Korea Tel: +82-2-2228-2443, Fax: +82-2-313-0956

E-mail: kimhs54@yuhs.ac

https://orcid.org/0000-0001-5662-7740 


\section{Study Highlights}

Wisteria floribunda agglutinin-positive human Mac-2 binding protein (WFA+-M2BP) level is an independent predictive factor of hepatitis B virus-related posthepatectomy carcinoma recurrence after curative resection. However, we found that WFA+-M2BP level was not predictive of grade B-C posthepatecomy liver failure or major complications. Patients with WFA ${ }^{+}-M 2 B P$ levels $>2.14$ were at significantly higher risk of overall recurrence and early recurrence than patients with WFA+-M2BP levels $\leq 2.14$. Further studies are needed to confirm whether current strategies used to select optimal candidates for resection can be optimized according to preoperative WFA+-M2BP values.

\section{INTRODUCTION}

If hepatocellular carcinoma (HCC) is detected in an early stage during surveillance, hepatic resection is strongly recommended for non-cirrhotic patients and is considered the mainstay of treatment in cirrhotic patients with well-preserved liver function.' Although recent advances in the selection of candidates for resection, optimization of surgical techniques, and perioperative management have improved the postoperative outcomes of patients with HCC, recurrence remains the main cause of an unsatisfactory long-term prognosis after HCC resection. ${ }^{2}$ Thus, identification of risk factors for recurrence after curative resection is of paramount importance.

Postoperative recurrence can be divided into early ( $<2$ years) and late ( $\geq 2$ years) recurrence, which have different underlying mechanisms. ${ }^{3}$ Advanced liver fibrosis or cirrhosis is a risk factor for late recurrence, as it contributes to multicentric recurrence in the remnant liver. ${ }^{4}$ In addition, advanced liver fibrosis or cirrhosis is closely correlated with short-term poor postoperative outcomes such as postoperative mortality or complications (e.g., liver failure and hepatic decompensation). ${ }^{5,6}$ Thus, preoperative assessment of the fibrotic burden can improve long-term outcomes by enabling selection of patients who will benefit from curative resection. Alternatively, other treatment modalities, such as liver transplantation, can be recommended to patients at greater risk for postoperative recurrence after curative resection.

Efforts have been made to expand the role of noninvasive surrogates for liver biopsy into the surgical setting, beyond simple assessment of the fibrotic burden. Indeed, preoperative liver stiffness values determined by transient elastography are reportedly predictive of the risk of the development of hepatic insufficiency, recurrence, and survival after curative HCC resection. ${ }^{7-9}$ Recently, a glycan-based immunoassay has been introduced; the assay targets Wisteria floribunda agglutinin-positive human Mac-2 binding protein (WFA $-\mathrm{M} 2 \mathrm{BP}$ ) as a noninvasive biomarker of liver fibrosis and predictor of HCC. ${ }^{10,11}$ Although a recent Japanese study found that WFA ${ }^{+}-\mathrm{M} 2 \mathrm{BP}$ was predictive of recurrence and survival in patients with early-stage HCC who underwent curative resection, ${ }^{12}$ the clinical role of WFA+-M2BP in surgical settings is still unclear.

Therefore, we conducted this single-center, retrospective cohort study to investigate the longitudinal prognostic performance of WFA ${ }^{+}-M 2 B P$ in predicting HCC recurrence in patients with hepatitis $B$ virus (HBV)-related HCC who underwent curative resection.

\section{PATIENTS AND METHODS}

\section{Patients}

Between 2004 and 2015, 492 patients who underwent curative resection for HBV-related HCC were considered eligible. Chronic hepatitis B (CHB) was defined as persistence of serum HBV surface antigen for $>6$ months. The exclusion criteria were no or an insufficient stored serum sample for WFA+ ${ }^{+}$M2BP assays, serum sample collected after resection, insufficient clinical data for statistical analysis, positive resection margin for HCC, co-infection with hepatitis $C$ virus or HIV, and alcohol ingestion of $>40$ g/day for $>5$ years.

This study was performed in accordance with the ethical guidelines of the 1975 Declaration of Helsinki and was approved by the Institutional Review Board of Severance Hospital. Given its retrospective nature, written informed consent for clinical data was not required.

\section{$\mathrm{WFA}^{+}-\mathrm{M} 2 \mathrm{BP}$ assay}

Serum WFA+-M2BP was quantified preoperatively through a lectin-Ab sandwich immunoassay using a fully automated immunoanalyzer (HISCL-2000i; Sysmex Co, Hyogo, Japan)..$^{13}$ For WFA+ M2BP assays, the remaining serum samples after HBV DNA quantification during the study period which have been stored at $-70^{\circ} \mathrm{C}$, were used. Measured levels of analyte conjugated with Wisteria floribunda agglutinin were indexed using the following equation:

Cutoff index $(\mathrm{COI})=\left(\left[\mathrm{WFA} 1^{+}-\mathrm{M} 2 \mathrm{BP}\right]_{\text {sample }}-\left[\mathrm{WFA} 1^{+}-\mathrm{M} 2 \mathrm{BP}\right]_{\mathrm{NC}}\right) /$ 
$\left(\left[W F A 1^{+}-M 2 B P\right]_{P C}\right)-\left[W F A 1^{+}-M 2 B P\right]_{N C}$

Where $\left[W F A 1^{+}-M 2 B P\right]_{\text {sample }}$ is the serum WFA ${ }^{+}-M 2 B P$ level, $P C$ is the positive control, and NC is the negative control. The positive control was supplied as a calibration solution preliminarily standardized to yield a COI of 1.0 .

\section{Preoperative work-up, surgery, and follow-up}

Diagnosis of HCC was based on the guidelines proposed by the Korea Liver Cancer Study Group. ${ }^{14}$ Preoperative HCC staging was assessed using the Barcelona Clinic Liver Cancer (BCLC) staging system. ${ }^{15}$ During follow-up, antiviral therapy was administered in accordance with the guidelines of the Korean Association for the Study of the Liver. ${ }^{16}$

As described in our previous study, ${ }^{7}$ all resections were performed by four experienced surgeons (DH Han, GH Choi, KS Kim, and JS (hoi). The type and extent of resections were determined according to tumor size, location, and liver reserve function estimated by the Child-Pugh score and indocyanine green retention rate at 15 minutes (ICG R15). Liver resection was defined as minor when two or fewer liver segments were resected, whereas major hepatectomy was defined as the resection of three or more liver segments. Intraoperative ultrasonography was performed routinely to determine tumor location and extent and to exclude the presence of additional lesions in the residual liver. Curative resection was defined as a negative pathological surgical margin and the absence of residual tumor. After discharge, patients were followed up every 3 months with tumor markers (alpha-fetoprotein [AFP] and des-gamma carboxy prothrombin [DCP]) and imaging studies, including abdominal computed tomography or magnetic resonance imaging.

\section{Definition of outcomes}

Posthepatectomy liver failure (PHLF) was defined and graded by the recommendations of the International Study Group of Liver Surgery (ISGLS). ${ }^{17}$ PHLF is defined as an increased international normalized ratio (INR) and hyperbilirubinemia on or after postoperative day 5. Grade A PHLF does not require deviations from regular clinical management, whereas grades B and C PHLF require noninvasive (grade B) or invasive treatment (grade C). If the INR or serum bilirubin concentration is increased preoperatively, PHLF is defined as an increasing serum bilirubin concentration on or after postoperative day 5 (compared to the values of the previous day). Postoperative complications were categorized according to the modified Clavien-Dindo classification (Supplementary Table 1). ${ }^{18}$ A major complication was defined as grade 3 or above. Recurrence was diagnosed based on the combined findings of the clinical examinations and sub-classified as early ( $<2$ years) or late $(\geq 2$ years) recurrence. ${ }^{3}$

\section{Histological assessment of tumor and non-tumor liver tissue}

As described in our previous study, ${ }^{7}$ a histological evaluation of the extracted liver specimens was performed by one experienced pathologist (YN Park) who was blinded to the patients' clinical information. Gross tumor classification, tumor size and number, vascular invasion, satellite nodule, and Edmondson-Steiner grade were determined. Liver fibrosis was evaluated semiquantitatively in non-cancerous tissues according to the Batts system. ${ }^{19}$ Fibrosis was staged on a $0-4$ scale: F0, no fibrosis; F1, portal fibrosis without septa; F2, portal fibrosis and a few septa; $F 3$, numerous septa without cirrhosis; and F4, cirrhosis.

\section{Statistical analysis}

Data are expressed as medians (interquartile range [IQR]) or $\mathrm{n}$ $(\%)$, as appropriate. Differences among continuous and categorical variables were examined using Student's $t$-tests (or MannWhitney tests) and chi-square tests (or Fisher's exact tests), respectively.

For longitudinal analysis, patients were followed up until the date of HCC recurrence or the last visit. Cumulative HCC recurrence rates were estimated using the Kaplan-Meier method and compared using log-rank tests. To identify independent risk factors for recurrence, univariate and multivariate Cox regression analyses were performed. In analyses of predictors of early recurrence ( $<2$ years), the analytic population included patients without recurrence during the study period, patients with recurrence after 2 years who were considered to not have had recurrence until the time of recurrence and censored at the same time, and patients with recurrence within 2 years. In analyses of predictors of late recurrence ( $\geq 2$ years), the analytic population included patients without recurrence, only if their follow-up period was more than 2 years, and patients with recurrence irrespective of followup time. To identify independent risk factors for developing grade B-C PHLF, univariate and multivariate binary regression analyses were performed. Hazard ratios (HRs) and corresponding 95\% 
confidence intervals (CIs) were calculated.

The diagnostic performance of WFA ${ }^{+}-M 2 B P$ was determined using receiver operating characteristic $(\mathrm{ROC})$ curves and the area under ROC curve (AUC). In addition, the cutoff values for predicting recurrence that maximized the sum of sensitivity (Se) and specificity (Sp) $\left(\right.$ Cutoff $\left._{\text {setsp }}\right)$, the cutoff values that showed positive predictive value (PPV) $>80 \%$ (Cutoff $_{\text {high-risk), and the cutoff values }}$ that showed negative predictive value (NVP) $>80 \%\left(\right.$ (Utoff $_{\text {low-risk }}$ ) were also calculated. All statistical analyses were performed using SPSS software (v20.0; IBM Corp., Armonk, NY, USA). A P-value $<0.05$ in two-tailed tests was considered to indicate statistical significance.

\section{RESULTS}

\section{Baseline characteristics}

After excluding 322 patients according to the exclusion criteria,

Table 1. Baseline characteristics of the study population $(n=170)$

\begin{tabular}{lc}
\hline Variable & Value \\
\hline Demographical variables & \\
Age (years) & $55(51-61)$ \\
Male sex & $132(77.6)$ \\
Body mass index $\left(\mathrm{kg} / \mathrm{m}^{2}\right)$ & $23.6(21.8-25.5)$ \\
Child-Pugh class A/B & $168(98.8) / 2(1.2)$ \\
Laboratory variables & \\
Aspartate aminotransferase (IU/L) & $27(23-35)$ \\
Alanine aminotransferase (IU/L) & $28(19-43)$ \\
Platelet count (10\%/L) & $157(126-200)$ \\
HBV-DNA levels (logio IU/mL) & $2.26(1.00-3.99)$ \\
Alpha-fetoprotein (ng/mL) & $13.0(3.3-97.5)$ \\
Des-gamma carboxyprothrombin & $44(26-188)$ \\
(mAU/mL) & \\
ICG R15 (\%) & $8.4(6.3-11.8)$ \\
WFA $+M 2 B P(C O I)$ & $1.02(0.70-1.55)$
\end{tabular}

Tumor stage

BCLC stage 0/A/B

$41(24.1) / 122(71.8) / 7(4.1)$

On-going antiviral therapy $126(74.1)$

Variables are expressed as median (interquartile range) or $n(\%)$. $\mathrm{HBV}$, hepatitis $B$ virus; ICG R15, indocyanine green retention rate at 15 minutes; WFA ${ }^{+}$-M2BP, Wisteria floribunda agglutinin-positive human Mac-2 binding protein; BCLC, Barcelona Clinic Liver Cancer.
170 patients with HBV-related HCC were selected for statistical analyses (Supplementary Fig. 1). The baseline characteristics of the study population before curative resection are summarized in Table 1. The median age of the entire cohort (132 males and 38 females) was 55 years. Most patients had well-preserved liver function of Child-Pugh class A ( $n=168,98.8 \%$ ).

The median AFP, DCP, ICG R15, and WFA ${ }^{+}$-M2BP levels were $13.0 \mathrm{ng} / \mathrm{mL}, 44 \mathrm{mAU} / \mathrm{mL}, 8.4 \%$, and $1.02 \mathrm{COI}$, respectively. $\mathrm{HCC}$ in BCLC stage 0 and A were identified in $41(24.1 \%)$ and 122 $(71.8 \%)$ patients, respectively. Of the patients, $126(74.1 \%)$ were receiving antiviral therapy at the time of resection.

\section{Perioperative outcomes and pathological information}

Perioperative outcomes and pathological information are summarized in Table 2. A total of 51 (30.0\%) patients underwent major hepatectomy. Most patients received open surgery $(n=113$, $66.5 \%$ ). The median amount of blood loss was $250 \mathrm{~mL}$ and blood

Table 2. Perioperative outcomes and pathological characteristics

\begin{tabular}{lc}
\hline Variable & Value \\
\hline Operative variables & \\
Type of hepatectomy (major/minor) & $51(30.0) / 119(70.0)$ \\
$\begin{array}{l}\text { Surgical approach (open/laparascop- } \\
\text { ic/robot) }\end{array}$ & $113(66.5) / 46(27.1) / 11(6.5)$ \\
Blood loss (mL) & $250(125-513)$ \\
Transfusion & $12(7.1)$ \\
Postoperative variables & \\
PHLF grading (0-A/B-C) & $158(95.2) / 8(4.8)$ \\
Complications (I-II/IIla-b/IV-V) & $163(95.9) / 7(4.1) / 0(0)$ \\
Tumor pathology & $2.4(1.9-3.2)$ \\
Maximal tumor size (cm) & $152(89.4) / 18(10.6)$ \\
Single/multiple tumors & $15(8.9)$ \\
Portal vein invasion & $78(46.2)$ \\
Microscopic vascular invasion & $12(7.1)$ \\
Satellite nodule & $91(55.2)$ \\
Edmondson-Steiner grade III-V & $71(41.8) / 99(58.2)$ \\
Non-tumor pathology & $162(95.3) / 8(4.7)$ \\
F0-3/F4 fibrosis & \\
A1-2/A3 activity grade & \\
\hline
\end{tabular}

Variables are expressed as median (interquartile range) or $n(\%)$. PHLF, posthepatectomy liver failure. 
transfusion was required in 12 (7.1\%) patients. Grade B-C PHLF developed in eight (4.8\%) patients. Seven (4.1\%) patients experienced major postoperative complications; the most common was pleural effusion $(n=4)$. In addition, bile leakage, ascites, and an umbilical incisional hernia developed in one patient each.

The median maximal tumor size was $2.4 \mathrm{~cm}$, and 18 (10.6\%) patients had multiple tumors. Portal vein invasion, microscopic vascular invasion, and satellite nodules were identified in 15 $(8.9 \%), 78(46.2 \%)$, and $12(7.1 \%)$ patients, respectively. Regarding non-tumor pathology, $99(58.2 \%)$ patients had F4 fibrosis. The WFA ${ }^{+}-M 2 B P$ level was significantly higher in patients with F4 than those with $\mathrm{FO}-3$ fibrosis (median 1.18 [IQR 0.79-1.86] vs. $0.91[0.67-1.31] ; P=0.045)$.

\section{Comparison between patients with and without HCC recurrence}

During the follow-up period (median 22.6 [IQR 13.3-61.0] months), $\mathrm{HCC}$ recurred in 64 (37.6\%) patients. The cumulative incidence of $\mathrm{HCC}$ recurrence at $1,2,3,4$, and 5 years was $15.8 \%$, $25.3 \%, 30.0 \%, 32.9 \%$, and $34.1 \%$, respectively (Supplementary Fig. 2). The most common site of recurrence was the liver $(n=58$, $90.6 \%)$.

A comparison of baseline characteristics between patients who developed recurrence and those who did not is shown in Table 3. Patients who developed recurrence had a higher HBV-DNA level (median 2.92 vs. $2.16 \log _{10} \mathrm{IU} / \mathrm{mL}$ ), higher WFA+ - M2BP level (median 1.22 vs. $0.91, \mathrm{COI}$ ), higher blood loss (median 330 vs. 250 $\mathrm{mL}$ ), greater maximal tumor size (median 2.6 vs. $2.2 \mathrm{~cm}$ ), and higher proportions of portal vein invasion (17.5 vs. $3.8 \%$ ), microscopic vascular invasion ( 58.7 vs. $38.7 \%$ ), and satellite nodules (12.9 vs. $3.8 \%)$ than patients who did not experience recurrence (all $P<0.05)$.

\section{Independent predictors of overall, early, and late recurrence}

Among the 64 patients with recurrence, 43 (67.2\%) experienced early recurrence and 21 (32.8\%) experienced late recurrence. We evaluated independent predictors of overall, early, and late recurrence (Table 4).

In univariate analyses, male sex, AFP level, DCP level, WFA'M2BP level, maximal tumor size, multiple tumors, portal vein invasion, microscopic vascular invasion, and the presence of satellite nodules were significantly predictive of overall, early, and late recurrence (all $P<0.05$ ), with the exception of borderline significance between multiple tumors and early recurrence $(P=0.051)$. In multivariate analyses, WFA ${ }^{+}-\mathrm{M} 2 \mathrm{BP}$ was an independent predictor of overall, early, and late recurrence (HR=1.490, 1.667, and 1.416, respectively; all $P<0.05$ ), together with other factors such as male sex, DCP, maximal tumor size, portal vein invasion, and satellite nodules.

\section{Independent predictor of grade B-C PHLF}

Univariate analyses identified aspartate aminotransferase level and major hepatectomy as significantly predictive of grade $B-C$ PHLF (all $P<0.05$ ). In subsequent multivariate analyses, major hepatectomy was the only independent predictor of grade B-C PHLF (HR $=4.254,95 \%$ Cl 1.824-9.919; $P=0.001$ ) (Supplementary Table 2).

\section{Optimal cutoff values of WFA ${ }^{+}-M 2 B P$ to predict recurrence}

Considering the median follow-up period (22.6 months), cutoff values for predicting 2-year recurrence in the whole study population and several subgroups were calculated based on ROC curve analyses (Table 5).

The WFA ${ }^{+}-M 2 B P$ cutoff value that maximized the sum of sensitivity (30.2\%) and specificity (90.6\%) was $2.14 \mathrm{COI}$ (AUC $=0.632$, $P=0.010$ ). Patients with a WFA ${ }^{+}-\mathrm{M} 2 \mathrm{BP}$ level $>2.14 \mathrm{CO}$ had a significantly higher cumulative recurrence rate than those with a $W_{F A}^{+}$-M2BP level $\leq 2.14 \mathrm{COI}(P=0.011$ by log-rank test) (Fig. 1). The cumulative incidences of HCC recurrence at 1, 2, 3, 4, and 5 years in patients with WFA ${ }^{+}$M2BP levels $>2.14 \mathrm{COI}$ were $32.0 \%$, $52.0 \%, 56.0 \%, 56.0 \%$, and $56.0 \%$, respectively, whereas those in patients with WFA ${ }^{+}$-M2BP levels $\leq 2.14 \mathrm{CO}$ were $13.1 \%$, $20.7 \%, 25.5 \%, 29.0 \%$, and $30.0 \%$, respectively. Using cutoff values for low and high risks of recurrence of $0.57 \mathrm{COI}$ (negative predictive value 89.4) and 3.88 (PPV 89.9\%), respectively (Table 5), only $1(6.2 \%)$ of 16 patients with a low risk of recurrence experienced recurrence, whereas $5(83.3 \%)$ of 6 patients with a high risk of recurrence experienced recurrence.

In patients with histological cirrhosis ( $n=99,58.2 \%$ ), the cutoff value of WFA ${ }^{+}$-M2BP that maximized the sum of sensitivity $(88.9 \%)$ and specificity (50.0\%) was $0.94 \mathrm{COI}$ (AUC $=0.720$, $P=0.001$ ), compared to $2.31 \mathrm{COI}$ (sensitivity $45.5 \%$ and specificity $97.5 \%, A \cup C=0.706, P=0.038)$ in patients who had undergone major hepatectomy $(n=51,30.0 \%)$. 
Table 3. Comparison between patients who did and did not experience hepatocellular carcinoma (HCC) recurrence

\begin{tabular}{|c|c|c|c|}
\hline Variable & $\begin{array}{l}\text { Patients who developed } \\
\text { HCC recurrence } \\
(n=64,37.6 \%)\end{array}$ & $\begin{array}{l}\text { Patients who did not develop } \\
\text { HCC recurrence } \\
(n=106,62.4 \%)\end{array}$ & $P$-value \\
\hline \multicolumn{4}{|l|}{ Demographical variables } \\
\hline Age (years) & $55(51-59)$ & $55(51-62)$ & 0.273 \\
\hline Male sex & $55(85.9)$ & 77 (72.6) & 0.057 \\
\hline Body mass index $\left(\mathrm{kg} / \mathrm{m}^{2}\right)$ & $23.3(21.6-25.9)$ & $23.6(21.9-25.0)$ & 0.766 \\
\hline Child-Pugh class A/B & $63(98.4) / 1(1.6)$ & $105(99.1) / 1(0.9)$ & 0.717 \\
\hline \multicolumn{4}{|l|}{ Laboratory variables } \\
\hline Aspartate aminotransferase (IU/L) & $28(24-39)$ & $27(22-34)$ & 0.814 \\
\hline Alanine aminotransferase (IU/L) & $30(20-43)$ & $27(18-43)$ & 0.739 \\
\hline Platelet count $\left(10^{9} / \mathrm{L}\right)$ & $164(126-204)$ & $153(236-196)$ & 0.626 \\
\hline HBV-DNA levels ( $\left.\log _{10} \mid \mathrm{I} / \mathrm{mL}\right)$ & $2.92(1.00-5.01)$ & $2.16(0.50-3.47)$ & 0.016 \\
\hline Alpha-fetoprotein (ng/mL) & $27.4(5.0-221.3)$ & $8.1(2.9-82.9)$ & 0.275 \\
\hline Des-gamma carboxyprothrombin (mAU/mL) & $55(27-239)$ & $40(26-134)$ & 0.341 \\
\hline ICG R15 (\%) & $9.4(6.3-12.2)$ & $8.0(6.3-11.7)$ & 0.924 \\
\hline $\mathrm{WFA}^{+}-\mathrm{M} 2 \mathrm{BP}(\mathrm{COI})$ & $1.22(0.79-1.79)$ & $0.91(0.68-1.41)$ & 0.017 \\
\hline \multicolumn{4}{|l|}{ Tumor stage } \\
\hline BCLC stage $0-A / B$ & $59(92.2) / 5(7.8)$ & $104(98.1) / 2(1.9)$ & 0.060 \\
\hline On-going antiviral therapy & $48(75.0)$ & $78(73.6)$ & 0.838 \\
\hline \multicolumn{4}{|l|}{ Operative variables } \\
\hline Type of hepatectomy (major/minor) & $49(76.6) / 15$ (23.4) & $36(34.0) / 70(66.0)$ & 0.147 \\
\hline Surgical approach (open/laparascopic+robot) & $42(65.6) / 22(34.3)$ & $71(67.0) / 35(33.0)$ & 0.868 \\
\hline Transfusion & $5(7.8)$ & $7(6.6)$ & 0.766 \\
\hline Blood loss (mL) & $330(150-750)$ & $250(100-450)$ & 0.018 \\
\hline \multicolumn{4}{|l|}{ Postoperative variables } \\
\hline PHLF grading (0-A/B-C) & $59(96.7) / 2(3.3)$ & $99(94.3) / 6(5.7)$ & 0.480 \\
\hline Complications (I-II/IIla-b) & $62(96.9) / 2(3.1)$ & $101(95.3) / 5(4.7)$ & 0.613 \\
\hline \multicolumn{4}{|l|}{ Tumor pathology } \\
\hline Maximal tumor size (cm) & $2.6(2.0-3.8)$ & $2.2(1.8-3.0)$ & 0.038 \\
\hline Multiple tumors & $10(15.6)$ & $8(7.5)$ & 0.097 \\
\hline Portal vein invasion & $11(17.5)$ & $4(3.8)$ & 0.003 \\
\hline Microscopic vascular invasion & $37(58.7)$ & $41(38.7)$ & 0.016 \\
\hline Satellite nodule & $8(12.9)$ & $4(3.8)$ & 0.033 \\
\hline Edmondson-Steiner grade III-V & $34(56.7)$ & $57(54.3)$ & 0.767 \\
\hline \multicolumn{4}{|l|}{ Non-tumor pathology } \\
\hline F4 fibrosis & $40(62.5)$ & $59(55.7)$ & 0.381 \\
\hline A3 activity grade & $40(62.5)$ & $5(4.7)$ & 0.993 \\
\hline
\end{tabular}

Variables are expressed as median (interquartile range) or $\mathrm{n}(\%)$ unless otherwise indicated.

HBV, hepatitis B virus; ICG R15, indocyanine green retention rate at 15 minutes; WFA+-M2BP, Wisteria floribunda agglutinin-positive human Mac-2 binding protein; BCLC, Barcelona Clinic Liver Cancer; PHLF, posthepatectomy liver failure. 


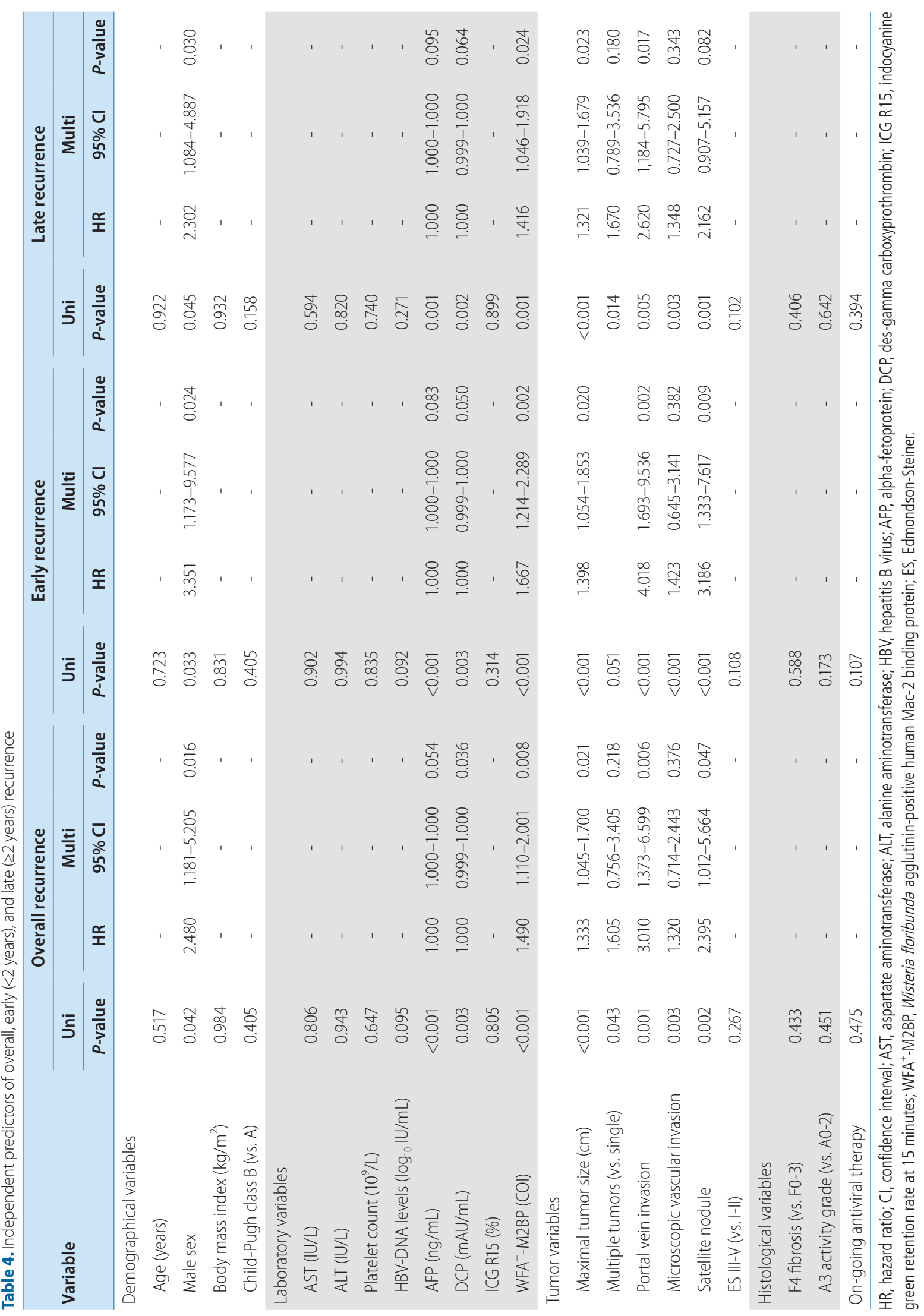


Table 5. WFA ${ }^{+}-M 2 B P$ cutoff values for 2-year recurrence and corresponding diagnostic indices

\begin{tabular}{|c|c|c|c|c|c|c|c|c|}
\hline Patient group & Cutoff values & AUC & $95 \% \mathrm{Cl}$ & $P$-value & Se (\%) & $\mathrm{Sp}(\%)$ & PPV (\%) & NPV (\%) \\
\hline Entire population & & 0.632 & $0.534-0.730$ & 0.010 & & & & \\
\hline Cutoff $_{\text {setsp }}$ & 2.14 & & & & 30.2 & 90.6 & 65.9 & 68.3 \\
\hline Cutoff $_{\text {low-risk }}$ & 0.57 & & & & 97.7 & 11.8 & 40.1 & 89.4 \\
\hline Cutoff high-risk $_{\text {}}$ & 3.88 & & & & 11.6 & 99.2 & 89.9 & 65.0 \\
\hline Histological cirrhosis & & 0.720 & $0.608-0.833$ & 0.001 & & & & \\
\hline Cutoff $_{\text {se }+ \text { sp }}$ & 0.94 & & & & 88.9 & 50.0 & 54.7 & 86.9 \\
\hline Cutoff $_{\text {low-risk }}$ & 0.94 & & & & 88.9 & 50.0 & 54.7 & 86.9 \\
\hline Cutoff $_{\text {high-risk }}$ & 3.88 & & & & 11.1 & 98.6 & 84.4 & 62.1 \\
\hline Major hepatectomy & & 0.706 & $0.513-0.898$ & 0.038 & & & & \\
\hline Cutoff $_{\text {se+sp }}$ & 2.31 & & & & 45.5 & 97.5 & 88.3 & 81.1 \\
\hline Cutoff low-risk $_{\text {. }}$ & 0.69 & & & & 90.9 & 37.5 & 37.7 & 90.8 \\
\hline Cutoff $_{\text {high-risk }}$ & 2.31 & & & & 45.5 & 97.5 & 88.3 & 81.1 \\
\hline
\end{tabular}

$\mathrm{WFA}^{+}-\mathrm{M} 2 \mathrm{BP}$, Wisteria floribunda agglutinin-positive human Mac-2 binding protein; AUC, area under receiver operating characteristic curve; $\mathrm{Cl}$, confidence interval; Se, sensitivity; Sp, specificity; PPV, positive predictive value; NPV, negative predictive value.

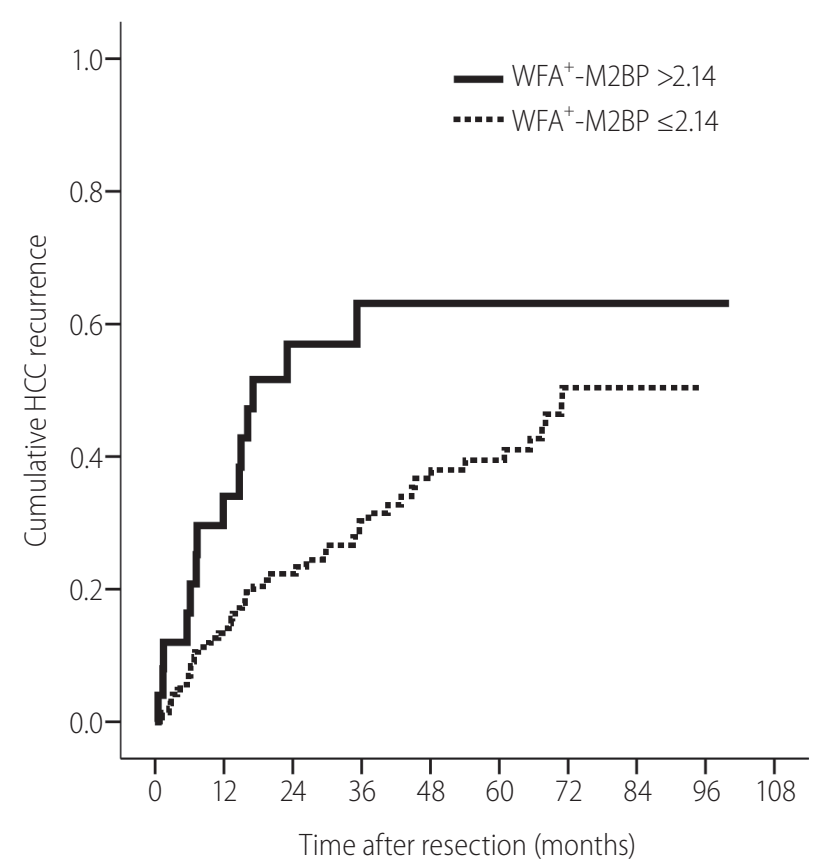

Figure 1. Cumulative incidence of hepatocellular carcinoma (HCC) recurrence according to WFA ${ }^{+}-M 2 B P$ level. The cumulative incidence increased significantly in patients with WFA-M2BP levels $>2.14$ than in those with WFA $-M 2 B P$ levels $\leq 2.14$ (log-rank test, $P=0.011$ ). WFA ${ }^{+}-M 2 B P$, Wisteria floribunda agglutinin-positive human Mac-2-binding protein.

\section{Postoperative outcomes according to WFA ${ }^{+}-M 2 B P$ cutoff value}

Postoperative outcomes according to preoperative WFA+-M2BP value are shown in Table 6. Patients with WFA ${ }^{+}-M 2 B P$ levels $>2.14(n=25,14.7 \%)$ had a significantly higher risk of overall recurrence $(56.0 \%$ vs. $34.5 \%)$ and early recurrence $(52.0 \%$ vs. $20.7 \%$ ) than patients with WFA ${ }^{+}-M 2 B P$ levels $\leq 2.14$ (all $P<0.05$ ). Although the risk of late recurrence was higher in patients with $W_{F A}^{+}-M 2 B P$ levels $>2.14$ compared to those with WFA ${ }^{+}-M 2 B P$ levels $\leq 2.14$, the association was of marginal significance $(P=0.072)$. The risks of grade B-C PHLF and major complications were statistically similar (all $P>0.05$ ) (Table 6).

\section{DISCUSSION}

Serum levels of WFA ${ }^{+}-M 2 B P$ may be useful as a marker of the degree of liver fibrosis. WFA ${ }^{+}-\mathrm{M} 2 \mathrm{BP}$ is secreted by hepatic stellate cells and enhances synthesis of Mac-2 by Kupffer cells, which renders hepatic stellate cells fibrogenic. ${ }^{20}$ WFA ${ }^{+}$-M2BP is a "doughnut-shaped" glycoprotein, changes in the $N$-glycan structure of which mirror the extent of liver fibrosis. ${ }^{21}$ Automated rapid assays for these changes have been developed for noninvasive assessment of fibrotic burden. Indeed, the diagnostic accuracy of WFA ${ }^{+}$M2BP for assessing the degree of liver fibrosis has been demonstrated in patients with $\mathrm{CHB}$, chronic hepatitis $\mathrm{C}$, non-alcoholic fatty liver disease, and biliary atresia. ${ }^{21-26}$ In addition, WFA ${ }^{+}-M 2 B P$ levels are predictive of the risk of development of HBV- and HCVrelated HCC. $^{11,23}$

Our study has several strengths. First, because the significant 
Table 6. Postoperative outcomes according to preoperative WFA ${ }^{+}-M 2 B P$

\begin{tabular}{|c|c|c|c|}
\hline Variable & $\begin{array}{l}\text { Patients with preoperative WFA }-M 2 B P \leq 2.14 \\
\qquad(n=145,85.3 \%)\end{array}$ & $\begin{array}{l}\text { Patients with preoperative WFA }{ }^{+}-M 2 B P>2.14 \\
\qquad(n=25,14.7 \%)\end{array}$ & $P$-value \\
\hline Overall recurrence & $50(34.5)$ & $14(56.0)$ & 0.047 \\
\hline Early recurrence & $30(20.7)$ & $13(52.0)$ & 0.001 \\
\hline Late recurrence & $20(13.8)$ & $1(4.0)$ & 0.072 \\
\hline Grade B-C PHLF & $6(4.1)$ & $2(8.0)$ & 0.420 \\
\hline Major complications & $5(3.4)$ & $2(8.0)$ & 0.290 \\
\hline
\end{tabular}

Variables are expressed as $n(\%)$ unless otherwise indicated.

WFA ${ }^{+}-M 2 B P$, Wisteria floribunda agglutinin-positive human Mac-2 binding protein; PHLF, posthepatectomy liver failure.

correlation between fibrotic burden assessed using WFA ${ }^{+}$-M2BP and the risk of $\mathrm{HCC}$ development was confirmed in previous studies, ${ }^{11,23}$ the ability of WFA - M2BP to predict the risk of HCC recurrence due to remnant fibrotic liver after curative resection should be investigated. Although serum levels of WFA+ $-M 2 B P$ measured at the time of curative resection are reportedly an independent predictor of $\mathrm{HCC}$ recurrence and survival in patients undergoing curative resection of $\mathrm{HCC}_{1}^{12}$ most of the patients in the study had HCV-related HCC (68.8\%)." Because the natural course of disease and the antiviral treatment strategy for HCV and HBV differ, it is important to validate the role of $\mathrm{WFA}^{+}-\mathrm{M} 2 \mathrm{BP}$ in patients with HBV-related HCC undergoing resection. The recent reports that $W_{F A}^{+}-M 2 B P$ levels are lower in patients with $C H B$ than in those with $\mathrm{CHC}^{22,27}$ also support the need for validation of WFA $-\mathrm{M} 2 \mathrm{BP}$ in patients with HBV-related HCC. To the best of our knowledge, this is the first report to focus on the prognostic value of WFA ${ }^{+}$M2BP for HBV-related HCC recurrence and survival in patients undergoing curative resection.

Second, we comprehensively investigated the role of WFA ${ }^{+}$$\mathrm{M} 2 \mathrm{BP}$ in predicting overall, early, and late recurrence as well as PHLF; the results suggested that WFA $-M 2 B P$ is predictive of all types of recurrence. Although early recurrence is generally associated with tumor factors, not those of the background liver, ${ }^{28}$ $W_{F A}^{+}-M 2 B P$ levels were independently associated with the risk of early recurrence in our study, together with several tumor factors such as AFP, DCP, maximal tumor size, portal vein invasion, and the presence of satellite nodules. However, the ability of WFA ${ }^{+}$$\mathrm{M} 2 \mathrm{BP}$ levels to predict early recurrence warrants further investigation.

Third, fibrosis stage (F0-3 vs. F4) was not predictive of recurrence in this study. This may be because the overall sample size was not sufficient to evaluate the prognostic accuracy of histological stage in terms of recurrence. In addition, more than half of the patients had histological cirrhosis (58.2\%). These factors might cause spectrum bias and potentially false-negative results. Also, it can be postulated that the linear expression of WFA M2BP with using the best cutoff values to predict recurrence might be better in predicting recurrence than histological information with step-wise crude expression. Furthermore, previous studies have reported other surrogates for liver fibrosis (such as transient elastography) to be predictive of HCC recurrence after curative resection in patients with $\mathrm{CHB}_{1}^{7,29}$ not histologic fibrosis stage. ${ }^{29}$ However, because the predictive accuracy of histological stage has been reported, ${ }^{4,28}$ our results warrant further investigation.

Fourth, we determined the optimal cutoff level of WFA ${ }^{+}-\mathrm{M} 2 \mathrm{BP}$ for predicting overall recurrence. This value (2.14) stratified subgroups with different risks of overall recurrence. Interestingly, our cutoff was lower than that of a previous study involving patients with HCV-related HCC undergoing curative resection (vs. 3.0).12 This is likely because over half of the study population had F4 stage fibrosis, and because HBV-related cirrhosis tends to be macronodular, leading to a lesser fibrotic burden than HCV-related cirrhosis. A lower cutoff WFA ${ }^{+}$-M2BP level in patients with HBV has been reported by others. ${ }^{27}$ In addition, a lower fibrotic burden in each fibrosis stage among patients with HBV-related chronic liver disease has been reported by previous studies of other surrogates for liver fibrosis (e.g., transient elastography). ${ }^{30}$ We also proposed clinically relevant cutoff values to enable identification of low- and high-risk patients. Only $6.2 \%$ of low-risk patients experienced recurrence, compared to $>80 \%$ of high-risk patients. These cutoff values might facilitate development of improved follow-up and management strategies.

This study also had several weaknesses. First, due to the insufficient sample size, a binary risk stratification of the study population using the calculated cutoff values was adopted. However, because a certain proportion of patients experienced recurrence of HCC despite a WFA ${ }^{+}-M 2 B P$ level lower than the cutoff value, fu- 
ture large-scale studies should involve multi-stage detailed risk stratification. Second, the effect of WFA ${ }^{+}-M 2 B P$ levels at the time of resection on overall survival could not be investigated because of the relatively short follow-up period (median, 22.6 months) and low mortality rate $(n=17,10.0 \%)$. Further studies with long-term follow-up are required to resolve this issue. Third, because this was a retrospective study and more than half of the participants were excluded, which might have caused selection bias, the results should be interpreted with caution. In addition, the distribution of each fibrosis stage was skewed (F1 in 1 [0.6\%] patient, F2 in 25 [14.7\%] patients, F3 in 45 [26.5\%] patients, and F4 in 99 [58.2\%] patients), which might resulted in the statistically nonsignificant prognostic value of F4 vs. F0-3. Probably due to the similar reason, fibrosis grade as an ordinal variable and different binary stratification such as F3-4 vs. F0-2 were significant neither. Finally, noninvasive surrogates for liver fibrosis, including WFA $^{+}-M 2 B P$ level, can predict the risk of developing PHLF after curative resection. ${ }^{31,32}$ However, we could not demonstrate that WFA ${ }^{+}$-M2BP was predictive of grade B-C PHLF in patients with HBV-related HCC undergoing curative resection. Major hepatectomy was the only independent predictor of grade B-C PHLF in this study.

In conclusion, our findings indicate a significant association between WFA ${ }^{+}-M 2 B P$ level and the risk of HBV-related HCC recurrence after curative resection in this population. However, it should be validated in further studies whether current strategies to select optimal candidates for resection and to evaluate the risk of postoperative recurrence can be optimized according to preoperative $W_{F A}^{+}-M 2 B P$ values.

\section{Authors' contribution}

The study was designed, directed and coordinated by Seung Up Kim. Gi Hong Choi and Hyon-Suk Kim, as the principal investigator, provided conceptual and technical guidance for all aspects of the project. Hyon-Suk Kim performed and analyzed WFA ${ }^{+}$-M2BP assays. Young Nyun Park provided histologic analysis from the specimen of curative resection of HCC. Dai Hoon Han, Kyung Sik Kim, Jin Sub Choi, Gi Hong Choi performed and graded perioperative outcomes and posthepatectomy liver failures. Hye Soo Kim, Beom Kyung Kim, Jun Yong Park, Do Young Kim, Sang Hoon Ahn, Kwang-Hyub Han contributed to the statistical analysis of patient survival. The manuscript was written by Seung Up Kim and Hye Soo Kim and commented on by all authors. All authors approved the final version of manuscript.

\section{Acknowledgements}

Graphics: The authors are grateful to Dong-Su Jang, (Medical IIlustrator, Medical Research Support Section, Yonsei University College of Medicine, Seoul, Korea) for his help with the figures.

Writing assistance: The English in this document has been checked by at least two professional editors, both native speakers of English (www.textcheck.com). For a certificate, please see:

http://www.textcheck.com/certificate/ESeGrC.

\section{Financial support}

This study was supported by Basic Science Research Program through the National Research Foundation of Korea funded by the Ministry of Science, Information and Communications Technology (ICT), and Future Planning (2016R1A1A1A05005138). The funders had no role in study design, data collection and analysis, decision to publish, or preparation of the manuscript.

\section{Conflicts of Interest}

None to declare for all authors.

\section{SUPPLEMENTARY MATERIALS}

Supplementary materials are available at Clinical and Molecular Hepatology website (http://www.e-cmh.org).

\section{REFERENCES}

1. Yu SJ. A concise review of updated guidelines regarding the management of hepatocellular carcinoma around the world: 2010-2016. Clin Mol Hepatol 2016;22:7-17.

2. Han DH, Choi GH, Park JY, Ahn SH, Kim KS, Choi JS, et al. Lesson from 610 liver resections of hepatocellular carcinoma in a single center over 10 years. World J Surg Oncol 2014;12:192.

3. Imamura H, Matsuyama Y, Tanaka E, Ohkubo T, Hasegawa K, Miyagawa $S$, et al. Risk factors contributing to early and late phase intrahepatic recurrence of hepatocellular carcinoma after hepatectomy. J Hepatol 2003;38:200-207.

4. Poon RT, Fan ST, Ng IO, Lo CM, Liu CL, Wong J. Different risk factors and prognosis for early and late intrahepatic recurrence after resection of hepatocellular carcinoma. Cancer 2000;89:500-507.

5. Kim SU, Ahn SH, Park JY, Kim DY, Chon CY, Choi JS, et al. Prediction of postoperative hepatic insufficiency by liver stiffness measurement (FibroScan((R))) before curative resection of hepatocellular carcinoma: a pilot study. Hepatol Int 2008;2:471-477. 
6. Shen Y, Zhou C, Zhu G, Shi G, Zhu X, Huang C, et al. Liver stiffness assessed by shear wave elastography predicts postoperative liver failure in patients with hepatocellular carcinoma. J Gastrointest Surg 2017:21:1471-1479.

7. Jung KS, Kim JH, Kim SU, Song K, Kim BK, Park JY, et al. Liver stiffness value-based risk estimation of late recurrence after curative resection of hepatocellular carcinoma: development and validation of a predictive model. PloS One 2014;9:e99167.

8. Kim SU, Kim BK, Han KH. Clinical application of liver stiffness measurement using transient elastography: a surgical perspective. Digestion 2013;88:258-265.

9. Cescon M, Colecchia A, Cucchetti A, Peri E, Montrone L, Ercolani G, et al. Value of transient elastography measured with FibroScan in predicting the outcome of hepatic resection for hepatocellular carcinoma. Ann Surg 2012;256:706-712; discussion 712-713.

10. Heo JY, Kim SU, Kim BK, Park JY, Kim DY, Ahn SH, et al. Use of Wisteria floribunda agglutinin-positive human Mac-2 binding protein in assessing risk of hepatocellular carcinoma due to hepatitis B virus. Medicine (Baltimore) 2016;95:e3328.

11. Kim SU, Heo JY, Kim BK, Park JY, Kim DY, Han KH, et al. Wisteria floribunda agglutinin-positive human Mac-2 binding protein predicts the risk of HBV-related liver cancer development. Liver Int 2017:37:879-887.

12. Toyoda H, Kumada T, Tada T, Kaneoka Y, Maeda A, Korenaga M, et al. Serum WFA+ -M2BP levels as a prognostic factor in patients with early hepatocellular carcinoma undergoing curative resection. Liver Int 2016;36:293-301.

13. Kuno A, Sato T, Shimazaki H, Unno S, Saitou K, Kiyohara K, et al. Reconstruction of a robust glycodiagnostic agent supported by multiple lectin-assisted glycan profiling. Proteomics Clin Appl 2013;7:642-647.

14. Korean Liver Cancer Study Group, National Cancer Center. Practice guidelines for management of hepatocellular carcinoma 2009. Korean J Hepatol 2009;15:391-423.

15. Kim BK, Kim SU, Park JY, Kim DY, Ahn SH, Park MS, et al. Applicability of BCLC stage for prognostic stratification in comparison with other staging systems: single centre experience from long-term clinical outcomes of 1717 treatment-naive patients with hepatocellular carcinoma. Liver Int 2012;32:1120-1127.

16. Korean Association for the Study of the Liver. KASL clinical practice guidelines: management of chronic hepatitis B. Clin Mol Hepatol 2012;18:109-162.

17. Rahbari NN, Garden OJ, Padbury R, Brooke-Smith M, Crawford $M$, Adam $R$, et al. Posthepatectomy liver failure: a definition and grading by the International Study Group of Liver Surgery (ISGLS). Surgery 2011;149:713-724.

18. Dindo D, Demartines N, Clavien PA. Classification of surgical complications: a new proposal with evaluation in a cohort of $6336 \mathrm{pa}-$ tients and results of a survey. Ann Surg 2004;240:205-213.

19. Batts KP, Ludwig J. Chronic hepatitis. An update on terminology and reporting. Am J Surg Pathol 1995;19:1409-1417.

20. Bekki Y, Yoshizumi T, Shimoda S, Itoh S, Harimoto N, Ikegami T, et al. Hepatic stellate cells secrete WFA+-M2BP: its role in biological interactions with Kupffer cells. J Gastroenterol Hepatol 2017;32:1387-1393.

21. Kuno A, Ikehara $Y$, Tanaka $Y$, Ito $K$, Matsuda A, Sekiya S, et al. A serum "sweet-doughnut" protein facilitates fibrosis evaluation and therapy assessment in patients with viral hepatitis. Sci Rep 2013;3:1065.

22. Zou X, Zhu MY, Yu DM, Li W, Zhang DH, Lu FJ, et al. Serum WFA+ -M2BP levels for evaluation of early stages of liver fibrosis in patients with chronic hepatitis B virus infection. Liver Int 2017;37:35-44.

23. Yamasaki K, Tateyama M, Abiru S, Komori A, Nagaoka S, Saeki A, et al. Elevated serum levels of Wisteria floribunda agglutinin-positive human Mac-2 binding protein predict the development of hepatocellular carcinoma in hepatitis C patients. Hepatology 2014;60:15631570.

24. Abe M, Miyake T, Kuno A, Imai Y, Sawai Y, Hino K, et al. Association between Wisteria floribunda agglutinin-positive Mac-2 binding protein and the fibrosis stage of non-alcoholic fatty liver disease. J Gastroenterol 2015;50:776-784.

25. Yamada N, Sanada Y, Tashiro M, Hirata Y, Okada N, Ihara Y, et al. Serum Mac-2 binding protein glycosylation isomer predicts grade F4 liver fibrosis in patients with biliary atresia. J Gastroenterol 2017; 52:245-252.

26. Fujiyoshi M, Kuno A, Gotoh M, Fukai M, Yokoo H, Kamachi $H$, et al. Clinicopathological characteristics and diagnostic performance of Wisteria floribunda agglutinin positive Mac-2-binding protein as a preoperative serum marker of liver fibrosis in hepatocellular carcinoma. J Gastroenterol 2015;50:1134-1144.

27. Nishikawa H, Enomoto H, Iwata Y, Kishino $K$, Shimono Y, Hasegawa $K$, et al. Serum Wisteria floribunda agglutinin-positive Mac-2-binding protein for patients with chronic hepatitis B and C: a comparative study. J Viral Hepat 2016;23:977-984.

28. Kim SU, Jung KS, Lee S, Park JY, Kim DY, Ahn SH, et al. Histological subclassification of cirrhosis can predict recurrence after curative resection of hepatocellular carcinoma. Liver Int 2014;34:1008-1017.

29. Jung KS, Kim SU, Choi GH, Park JY, Park YN, Kim DY, et al. Prediction of recurrence after curative resection of hepatocellular carcinoma using liver stiffness measurement (FibroScan(R)). Ann Surg Oncol 2012;19:4278-4286.

30. Seo YS, Kim MY, Kim SU, Hyun BS, Jang JY, Lee JW, et al. Accuracy of transient elastography in assessing liver fibrosis in chronic viral hepatitis: a multicentre, retrospective study. Liver Int 2015;35:22462255.

31. Okuda Y, Taura K, Yoshino K, Ikeno Y, Nishio T, Yamamoto G, et al. 


\section{CMLH}

Volume_26 Number_1 January 2020

Usefulness of Mac-2 binding protein glycosylation isomer for prediction of posthepatectomy liver failure in patients with hepatocellular carcinoma. Ann Surg 2017;265:1201-1208.

32. Chong CC, Wong GL, Chan AW, Wong VW, Fong AK, Cheung YS, et al. Liver stiffness measurement predicts high-grade post-hepatectomy liver failure: a prospective cohort study. J Gastroenterol Hepatol 2017:32:506-514. 